\title{
Investigando el estilo personal del terapeuta: Correspondencia entre las autodescripciones y la observación de jueces externos
}

\author{
Javier Fernández-Álvarez ${ }^{1,2}$, Claudia Castañeiras ${ }^{2}$, Fernando García ${ }^{2}$, Beatriz Gómez ${ }^{2}$ \\ y Héctor Fernández-Álvarez ${ }^{2}$ \\ ${ }^{1}$ Universidad Jaime I, Castellón, España \\ ${ }^{2}$ Fundación Aiglé, Buenos Aires, Argentina
}

Correspondence between self-descriptions and observations of external judges: A study on the personal style of the therapist

\begin{abstract}
The personal style of the therapist (PST) is defined as the set of characteristics that lead the therapist to behave in a particular professional way. This empirical study aims to provide evidence of the concurrent validity of the EPT-C, an instrument to assess PST. Cluster analysis was performed with data from 79 audio-taped recorded sessions of 20 psychotherapists. The contrasting groups' method was applied to compare EPT-C scores with the scores of the Guide for the Observation of the Therapist Aiglé (GOTA-v) as the criterion variable. The mean values, obtained in the EPT-C dimensions of three therapists' clusters arising from the sessions' observations and codification of emerging categories, were compared. Significant differences were found in two EPT-C dimensions: attentional and operative. These results are evidence of the concurrent validity of the EPT-C questionnaire.
\end{abstract}

Keywords: Personal style of the therapist; EPT-C; GOTA-v; concurrent validity.

Resumen: El estilo personal del terapeuta (EPT) se conceptualiza como el conjunto de condiciones singulares que conducen a un terapeuta a operar en su desempeño profesional. El objetivo de este estudio empírico es aportar evidencia sobre la validez concurrente de un instrumento que evalúa el EPT (EPT-C). Se realizó un análisis de cluster a partir de 79 sesiones transcriptas de 20 psicoterapeutas. Para ello, se aplicó metodología de grupos contrastados para comparar los puntajes del EPT-C con los valores de la Guía de Observación del Terapeuta Aiglé (GOTA-v) como variable criterio. Se compararon las medias obtenidas en las dimensiones EPT-C para tres clusters de terapeutas, identificados a partir de la observación de las sesiones y la codificación de las categorías emergentes. Se obtuvieron diferencias significativas en dos dimensiones del el EPT-C: la atencional y la operativa. Los resultados evidencian la validez concurrente del instrumento EPT-C para evaluar el EPT.

Palabras clave: Estilo Personal del Terapeuta, EPT-C, GOTA-v, Validez concurrente.

\section{Introducción}

Los hallazgos disponibles desde hace más de 3 décadas ponen de manifiesto que la psicoterapia constituye un método efectivo para el mejoramiento de la salud mental y la calidad de vida de las personas. El principal

Recibido: 01 febrero 2017; aceptado: 08 junio 2017

Correspondencia: Javier Fernández-Álvarez, Facultad de Psicología, Universidad Jaime I, Avda. Vicent Sos Baynat s/n, 12071 Castellón, España. Correo-e: fernanja@uji.es y sustancial aporte para ello se ha producido gracias a la proliferación de estudios controlados y aleatorizados que dieron lugar a la aparición de estudios meta-analíticos, permitiendo precisar la magnitud del efecto de la psicoterapia en el tratamiento de diversas condiciones clínicas y poblaciones (García-Escalera, Chorot, Valiente, Reales y Sandín, 2016; Seligman, 1995; Wampold y Imel, 2015).

La mayor parte de los estudios se han centrado en determinar la eficacia de tratamientos o ingredientes específicos, con la consecuente aparición de guías de trata- 
mientos y recomendaciones clínicas en distintas partes del mundo como las guías NICE del Servicio Nacional de Salud en Gran Bretaña, las guías de la Asociación Americana de Psicología o las guías del Servicio Nacional de Salud de España, así como recopilaciones bibliográficas que reúnen la evidencia principal para las diversas condiciones clínicas de manera concisa y exhaustiva (Nathan y Gorman, 2015; Roth y Fonagy, 2010). A pesar de que los avances descritos han sido necesarios y se destacan como fuente fiable de conocimiento, el estudio de otros factores terapéuticos, así como de los aspectos procesuales que permitirían precisar la incidencia de las variables mediadoras, moderadoras y los mecanismos de cambio, no ha contado con suficiente evidencia empírica (Wampold y Imel, 2015).

En la medida en que se asuma el desafío de avanzar hacia la personalización de los tratamientos en salud mental, será indispensable poder integrar los factores relegados, y las metodologías alternativas a los estudios aleatorizados de eficacia, que si bien han resultado y seguirán siendo valiosos, no carecen de problemas (Beutler y Forrester, 2014; Cuijpers, Ebert, Acarturk, Andersson y Cristea, 2016).

En esta dirección, el estudio de las variables del terapeuta cobra particular importancia, tanto en lo referido a la caracterización y descripción de perfiles terapéuticos como al estudio de la efectividad diferencial en relación al proceso y resultado en psicoterapia. Durante las últimas décadas ha habido diversos estudios al respecto que han considerado aspectos demográficos, patrones de personalidad, bienestar emocional, factores cognitivo-afectivos (p.ej. valores o creencias), atributos de influencia social, factores socio-profesionales y estilo terapéutico (Badlwin y Imel, 2013; Beutler et al., 2004; Crits-Cristoph y Mintzel, 1991).

El programa de investigación sobre el Estilo Personal del Terapeuta en la Fundación Aiglé tiene como objetivo principal el estudio de procesos y resultados en psicoterapia con el propósito de optimizar la práctica clínica en contextos de asistencia y entrenamiento de terapeutas (Fernández-Álvarez, 2008; Fernández-Álvarez, Gómez y García, 2015). De esta manera, se considera la complejidad inherente para aumentar la validez ecológica (Norcross y Lambert, 2014). Precisamente en los ámbitos clínicos reales, el efecto del terapeuta se puede ver mitigado en comparación a ámbitos de estudio controlados en los que se llevan a cabo tratamientos protocolizados. La principal razón que explica dicha diferencia es que los protocolos presentan un alto grado de estructuración formal y técnica, mientras que en contextos clínicos reales los aspectos inespecíficos cobran mayor protagonismo en la explicación del cambio terapéutico (Huppert et al., 2014).
En el marco de este programa general se vienen desarrollando estudios sobre el Estilo Personal del Terapeuta (EPT). El EPT ha sido definido como un constructo multidimensional que permite conceptualizar las características personales relativamente estables de los terapeutas, que modulan el modo en el que aplica las técnicas y procedimientos en sesión. Asimismo, se considera que el EPT actúa como un principio general de la psicoterapia, independientemente del tipo y modo de abordaje, de las características del paciente o de cualquier otra circunstancia específica, y que resulta una variable de impacto en los resultados terapéuticos (Fernández-Álvarez, García y Scherb, 1998).

En cuanto al desarrollo del constructo, se identificaron 5 dimensiones pertenecientes al conjunto de funciones en las que se basan las acciones del terapeuta. Estas 5 dimensiones, que al comienzo fueron 6 (ya que se incluía la dimensión evaluativa), surgieron de la experiencia clínica de los autores y del trabajo conjunto con Larry Beutler a partir de la Selección Sistemática de tratamiento (STS) (Beutler y Harwood, 2000). El primer paso consistió en desarrollar un cuestionario, para lo cual se generaron múltiples preguntas para su construcción y se lo administró a diferentes muestras piloto hasta llegar a la muestra que dio lugar a la forma actual. En esta línea, se realizaron distintos estudios con el EPT-C (García y Fernández-Álvarez, 2007). Su operacionalización dio lugar a un inventario autodescriptivo, el EPT-C, que consta de 36 ítems. El instrumento ha demostrado tener propiedades psicométricas satisfactorias. Por un lado, posee una adecuada consistencia interna (instruccional; alfa de Cronbach $=0,69$; expresiva; alfa de Cronbach $=0.75$; involucración; alfa de Cronbach $=0.75$; atencional; alfa de Cronbach $=0.80$; operativa; alfa de Cronbach $=0.79)($ Fernández-Álvarez et al., 2003) y estabilidad temporal (García y Fernández-Álvarez, 2007).

Además, se ha estudiado su validez de constructo a través de análisis factoriales exploratorios y confirmatorios en distintos grupos que indican una estructura relacionada pero independiente de las 5 dimensiones (Fernández-Álvarez, García, Lo Bianco y Corbella Santomá, 2003; Castañeiras, Ledesma, García y Fernández-Álvarez, 2008), con dos factores de segundo orden: uno que integra los aspectos técnicos del EPT incluyendo las funciones atencional y operativa, y otro que agrupa las funciones expresiva, de involucración e instruccional en torno al componente relacional de la psicoterapia (Castañeiras et al., 2008).

Las cinco dimensiones del instrumento son: (1) Instruccional: Responde a la instalación y ajuste del dispositivo terapéutico. Los terapeutas varían entre rígidos y flexibles. Ejemplos de reactivos son: «Me considero un 
terapeuta que trabaja con un encuadre flexible» $\mathrm{y}$ «Tiendo a exigir cumplimiento estricto con los honorarios»; (2) Expresiva: Hace referencia al modo de relacionarse afectivamente con el paciente. Los terapeutas se ubican en alguna posición en cuanto a la distancia emocional (próximos-distantes). Ejemplos de reactivos: «Evito comunicarme a través de gestos y expresiones muy emotivas» $\mathrm{y}$ «La expresión de las emociones es un poderoso instrumento de cambio»; (3) Involucración: Describe el grado en el que los terapeutas se involucran en la tarea (muy comprometidos - poco comprometidos). Ejemplos de reactivos: «Pienso bastante en mi trabajo aún en mis horas libres» $\mathrm{y}$ «Lo que les pasa a mis pacientes tiene poca influencia en mi vida personal»; (4) Atencional: Se refiere al despliegue de los elementos necesarios para avanzar en el trabajo terapéutico, que puede variar entre focalizados/activos, y abiertos/receptivos. Ejemplos de reactivos: «Desde el comienzo de las sesiones me dispongo a dejar flotar mi atención» y «Me interesa trabajar con pacientes que presentan problemas focalizados»; (5) Operativa: Responde al modo de cumplimiento de las tareas. Los terapeutas pautados presentarán un estilo más directivo y sujeto a reglas, mientras que los más espontáneos serán más intuitivos y no directivos. Ejemplos de reactivos: «En mis intervenciones soy predominantemente directivo» $\mathrm{y}$ 《Las mejores intervenciones en un tratamiento surgen de modo espontáneo».

Por otro lado, el EPT-C ha sido utilizado desde hace más de una década para la investigación empírica en estudios sobre la relación del estilo personal del terapeuta y los perfiles de los pacientes (Corbella, 2002), las características del EPT en la Selección Sistemática de Tratamientos (Malik et al., 2002; Corbella et al., 2003), la indagación de estilos según orientación teórica de los terapeutas (Vázquez y De Vázquez, 2015), y el estudio del EPT de terapeutas con diversas poblaciones clínicas (Casari, Albanesi, Maristany y Ison, 2016; Gómez, 2015; Rial, Castañeiras, García, Gómez y Fernández-Álvarez, 2006; Vega, 2006). Todas estas evidencias están basadas, sin embargo, en las autodescripciones de los terapeutas. Con el objetivo de establecer la validez concurrente se propuso utilizar metodología observacional para evaluar las conductas verbales de los terapeutas durante las sesiones.

Para ello se diseñó y protocolizó una guía observacional, la Guía Observacional de Terapeutas Aiglé (GOTA-v; Fernández-Álvarez, Gómez, Curtarelli, Lichtenberger y García, 2015), diseñada para su aplicación al estudio del estilo personal. A través de ella, jueces independientes pueden analizar la conducta verbal de los terapeutas durante las sesiones. Su elaboración tiene como antecedentes otros sistemas clasificatorios de las accio- nes verbales de los terapeutas (Roussos, Etchebarne y Waizmann, 2006; Stiles, 1992; Valdés, Tomicic, Pérez y Krause, 2010). La GOTA-v fue diseñada específicamente para estudiar estas conductas con independencia de los aspectos relacionales y los efectos de las intervenciones siguiendo principios gramaticales que permiten la delimitación de los actos de habla, cualquiera sea la orientación teórica del terapeuta (Searle, 1967). Su estructura establece tres tipos de intervenciones: preguntas, afirmaciones e indicaciones. Las dos primeras se desagregan en 6 y 8 categorías respectivamente, mientras que para las indicaciones se considera el aspecto estrictamente sintáctico del modo imperativo en una única categoría (Fernández-Álvarez et al., 2015). La aplicabilidad del instrumento para su uso en distintos contextos de investigación y aplicación ha dado lugar a índices $\mathrm{K}$ de kappa de $k=0.76$ para afirmaciones y $k=0.79$ para preguntas (Fernández-Álvarez et al., 2015).

En relación a la aplicación de la GOTA-v, los estudios realizados sobre el efecto moderador de la orientación teórica y el nivel de experiencia no informan diferencias significativas en los tipos de intervención ni en sus frecuencias con respecto a estas variables de comparación (Gómez et al., 2013; Gómez et al., 2014). Aunque es necesario profundizar su estudio en relación al tipo y cantidad de intervenciones y los momentos de la sesión en las que se realizan, el supuesto teórico subyacente considera que el EPT se trataría de una variable moderadora en cualquier orientación teórica o nivel de experiencia. Finalmente, el objetivo del estudio que se presenta es aportar evidencia sobre la validez concurrente de las dimensiones del EPT-C en función de los resultados obtenidos a partir de la aplicación de la GOTA-v.

Como en las categorías que aporta la GOTA-v se consideran específicamente aspectos verbales de las intervenciones de los terapeutas en sesión, se espera hallar asociaciones con dos de las cinco dimensiones que incluyen los reactivos del EPT-C: la atencional y la operativa, dado que son las referidas predominantemente a los aspectos verbales que con más frecuencia se presentan en las intervenciones de los terapeutas. Las otras tres dimensiones (instruccional, expresiva y de involucración) se estiman menos detectables mediante el análisis transcrito de las sesiones, por estar más vinculadas con aspectos relacionales y de la conducta del terapeuta fuera de la sesión, así como a otros aspectos para-verbales y no verbales que no contempla la GOTA-v. Por ejemplo, es mucho menos frecuente que el terapeuta se ocupe de los honorarios o del cumplimiento de los horarios. De igual modo que el terapeuta tenga presente a sus pacientes fuera de las sesiones se basa en la experiencia subjetiva del terapeuta. Por último, las preguntas sobre la dimen- 
sión expresividad recogen aspectos predominantemente no verbales del discurso durante las sesiones.

El objetivo general de este estudio es aportar evidencia de validez concurrente para el EPT. Para ello se exploraron las relaciones entre las dimensiones del EPT-c y las categorías de la GOTA-v en 20 terapeutas integrativos. A su vez te consideraron los siguientes objetivos específicos: (1) Identificar una estructura de clusters que permita agrupar los 20 terapeutas en función de las intervenciones verbales; (2) Establecer los perfiles de cada cluster en función de las diferencias significativas entre ellos; (3) Establecer si existen diferencias significativas en las puntuaciones del EPT-C entre los terapeutas de los diferentes clusters. En cuanto a las hipótesis, proponemos las siguientes: (1) Existen diferencias estadísticamente significativas en las puntuaciones de la dimensión atencional del EPT-C entre los terapeutas de los diferentes cluster surgidos de la clasificación de las intervenciones a través de la GOTA-v; (2) Existen diferencias estadísticamente significativas en las puntuaciones de la dimensión operativa del EPT-C entre los terapeutas de los diferentes cluster surgidos de la clasificación de las intervenciones a través de la GOTA-v.

\section{Método}

\section{Participantes}

Se analizaron 79 sesiones correspondientes a 20 terapeutas integrativos de baja, media y alta experiencia (rango etario: $28-70$ años; $M=47 ; D T=3$ ). Esto equivale a 4 sesiones por terapeuta, con excepción de un terapeuta del que solo se pudieron obtener 3 sesiones. Todos los tratamientos se realizaron en un contexto de práctica privada.

Los criterios de selección fueron los siguientes dos: en primer lugar, se incluyeron sesiones de pacientes con diagnóstico de trastorno emocional, trastorno adaptativo y/o de personalidad, indistintamente hombres o mujeres adultos y en tratamiento en dispositivo individual. Se excluyeron sesiones de pacientes con trastornos mentales graves y/o con abuso de sustancias. En segundo lugar, se utilizaron en todos los casos sesiones que se consideraron clínicamente más representativas del proceso psicoterapéutico, esto es: no de inicio del tratamiento en el que se instala el dispositivo terapéutico ni del final del tratamiento. Tampoco sesiones que presentaran características excepcionales, a juicio de los terapeutas, en cuanto al manejo y los contenidos de las intervenciones. Los terapeutas participantes, teniendo en cuenta estos criterios de exclusión proporcionaron voluntariamente las grabaciones de sus sesiones.

\section{Instrumentos}

Cuestionario de Evaluación del Estilo Personal del Terapeuta (EPT-C; Fernández-Álvarez y García, 1998). Cuestionario auto descriptivo de 36 ítems que se responden con una escala Likert de 7 puntos, desde totalmente en desacuerdo a totalmente de acuerdo. Los resultados expresan la posición de los terapeutas en 5 dimensiones que componen el Estilo Personal: instruccional, expresiva, involucración, atencional y operativa.

Guía para la Observación y Clasificación de la Conducta Verbal de los Terapeutas de Aiglé (GOTA-v; Fernández-Álvarez et al., 2015). Es una guía que permite clasificar las conductas verbales de los terapeutas en las sesiones, en términos de tres grandes categorías de actos de habla: preguntas, afirmaciones e indicaciones. Los resultados se expresan en frecuencias de intervenciones en esas categorías y sus diferentes subcategorías.

\section{Procedimiento}

En primer lugar, se convocó a 20 terapeutas integrativos (esta denominación se basó en la autodescripción de los terapeutas a esa orientación teniendo en cuenta los criterios mencionados) y se les informó del objetivo del estudio. Previa firma del consentimiento informado se procedió a que cada uno de los terapeutas completara el EPT-C. Posteriormente, se gestionó la recepción de 4 sesiones transcritas aportadas por cada terapeuta (una sesión por paciente). En todos los casos, también los pacientes firmaron un consentimiento informado autorizando la grabación de las respectivas sesiones y su participación en la investigación. La muestra consistió en terapeutas que conforman la Red de Terapeutas de la Fundación Aiglé (Buenos Aires, Argentina).

De cada sesión se seleccionaron y transcribieron los 10 minutos iniciales, los 10 minutos del medio y los 10 minutos finales, en función del cumplimiento de dos condiciones: a) la necesidad de homogeneizar las unidades de análisis (extensión de las sesiones), y b) delimitar los momentos que en sesión suelen resultar de mayor posibilidad de expresión del estilo de intervención del terapeuta. Este criterio no está exento de cuestionamientos. Sería ideal la transcripción de la totalidad de las sesiones. Sin embargo la secuencia de las sesiones admite un momento de apertura, un momento de desarrollo y uno de cierre. Luego de una revisión de cada transcripción para garantizar la fidelidad de las mismas, se identificaron las unidades de análisis de acuerdo a las reglas propuestas por Hill y O'Brien (1999), que permiten delimitar la totalidad del texto en cláusulas independientes, dependientes o subordinadas, así como establecer los 
criterios de exclusión para aquellas expresiones que no son cláusulas. Este procedimiento fue realizado por dos jueces independientes, y en el caso de discrepancias se contó con la participación de un tercer juez que definió la pertenencia a la categoría.

En un segundo momento la aplicación de la GOTA-v incluyó la clasificación de las unidades de análisis en función de las principales clases generales de actos de habla: preguntas, afirmaciones e indicaciones. Cada una de estas clases incluye subcategorías las cuales se delimitan en función del contenido y de la intencionalidad de las acciones verbales (ver Anexo I). De manera equivalente al procedimiento para las unidades de análisis, dos jueces independientes establecieron las categorías de intervenciones verbales para cada sesión, y un tercero resolvió las discrepancias. Aquellos que resolvieron discrepancias fueron en todos los casos los investigadores con más experiencia en la utilización del instrumento (segunda y tercera autor y cuarto autor) (Fernández-Álvarez et. al., 2015).

\section{Análisis estadístico de los datos}

Con el propósito de establecer perfiles de terapeutas de acuerdo a las intervenciones verbales identificadas a través de la GOTA-v, se realizó el análisis de cluster aplicado directamente a las categorías de la guía GOTA (preguntas, afirmaciones e indicaciones) con su mayor desagregación. Se utilizó el método jerárquico de clasificación de WARD por tratarse de la opción más estadística más apropiada de las disponibles, al basar el criterio de agrupamiento de los casos en la minimización de la varianza interna de los grupos conformados. Se decidió la utilización de este procedimiento debido a su alta interpretabilidad, su uso frecuente en las ciencias del comportamiento y su eficacia en la recuperación de la estructura subyacente. La distancia euclidiana cuadrada se utilizó como medida de similitud. Para determinar el número de clusters a considerar se utilizó el dendrograma que grafica el esquema de unión de los distintos elementos, de acuerdo a su grado de similitud.

De cada una de las variables que componen la GOTA-v se realizó un análisis de varianza ANOVA de un factor respecto a los 3 clusters identificados para establecer aquellas categorías en las que cada uno de los grupos se diferencia significativamente desde el punto de vista estadístico, con las comparaciones múltiples a posteriori (DHS Tukey) para determinar entre qué grupos se presentaban dichas diferencias.

A los efectos de analizar la posible relación entre las categorías de la GOTA-v y las puntuaciones del EPT-C se evaluó la presencia de diferencias con significación estadística en las puntuaciones medias del EPT-C de los terapeutas incluidos en cada cluster mediante ANOVA de un factor. Asimismo, se llevaron a cabo pruebas post hoc (Tukey) para determinar la fuente de diferencias observadas, calculándose posteriormente el tamaño del efecto eta al cuadrado parcial $\left(\eta_{\mathrm{p}}^{2}\right)$.

\section{Resultados}

En su conjunto, para las 79 sesiones la GOTA-v produjo una clasificación de 3.789 preguntas, 12.292 afirmaciones, 509 indicaciones y 338 actos sin clasificar, lo que equivale a un total de 16.928 unidades de análisis. A partir del análisis de cluster realizado se obtuvo una solución de tres grupos de terapeutas (clusters) con el procedimiento jerárquico detallado. Otras soluciones que incluyeran más de 3 clusters no resultaban adecuadas a la hora de reducir las aglomeraciones de los coeficientes o para mejorar la interpretabilidad de los clusters. En la Figura 1 se presenta el dendrograma correspondiente en el que se observan los 3 clusters seleccionados.

Cada uno de los clusters representa un perfil terapéutico. A continuación se presenta la frecuencia de categorías en relación a las unidades de análisis de cada cluster (Figura 2). Para interpretar el significado de las siglas que designan las categorías, ver Anexo I. Se detectaron diferencias estadísticamente significativas entre los clusters en algunas categorías. Concretamente, en cuanto a las preguntas en PHc $(F=5.889 ; p=0.011)$ y en PC $(F$ $=14.961 ; p=0.000)$. En relación al uso de afirmaciones durante la sesión, las diferencias se hallaron en $\mathrm{AI}(F=$ $14,397 ; p=0,000)$, en AR $(F=6.480 ; p=0.008)$, en AMA $(F=10.993 ; p=0.001)$, y en $\operatorname{AE}(F=4.567 ; p=$ 0.026).

En función de las comparaciones múltiples realizadas a partir de la prueba post-hoc DHS Tukey, es posible especificar que en relación a las preguntas cerradas (PHc) la diferencia se identifica entre el cluster 1 respecto al cluster 2 y al cluster 3, al igual que en las afirmaciones para dar información (AI) y en afirmaciones que retoman algo que dice el paciente (AR). En cuanto a las afirmaciones que van más allá de lo que dice el paciente (AMA) se ha encontrado una diferencia entre el cluster 1 y 3 así como entre el 2 y el 3. En cuanto a las las afirmaciones respecto al encuadre (AE) la diferencia observada ha sido únicamente entre el cluster 1 y el 3. Por último, respecto a las preguntas por confirmación (PC) hay diferencia entre todos los grupos.

Asimismo, en la tabla 1 se exponen los resultados del análisis de varianza (ANOVA) de un factor realizado para establecer las diferencias en las dimensiones del 


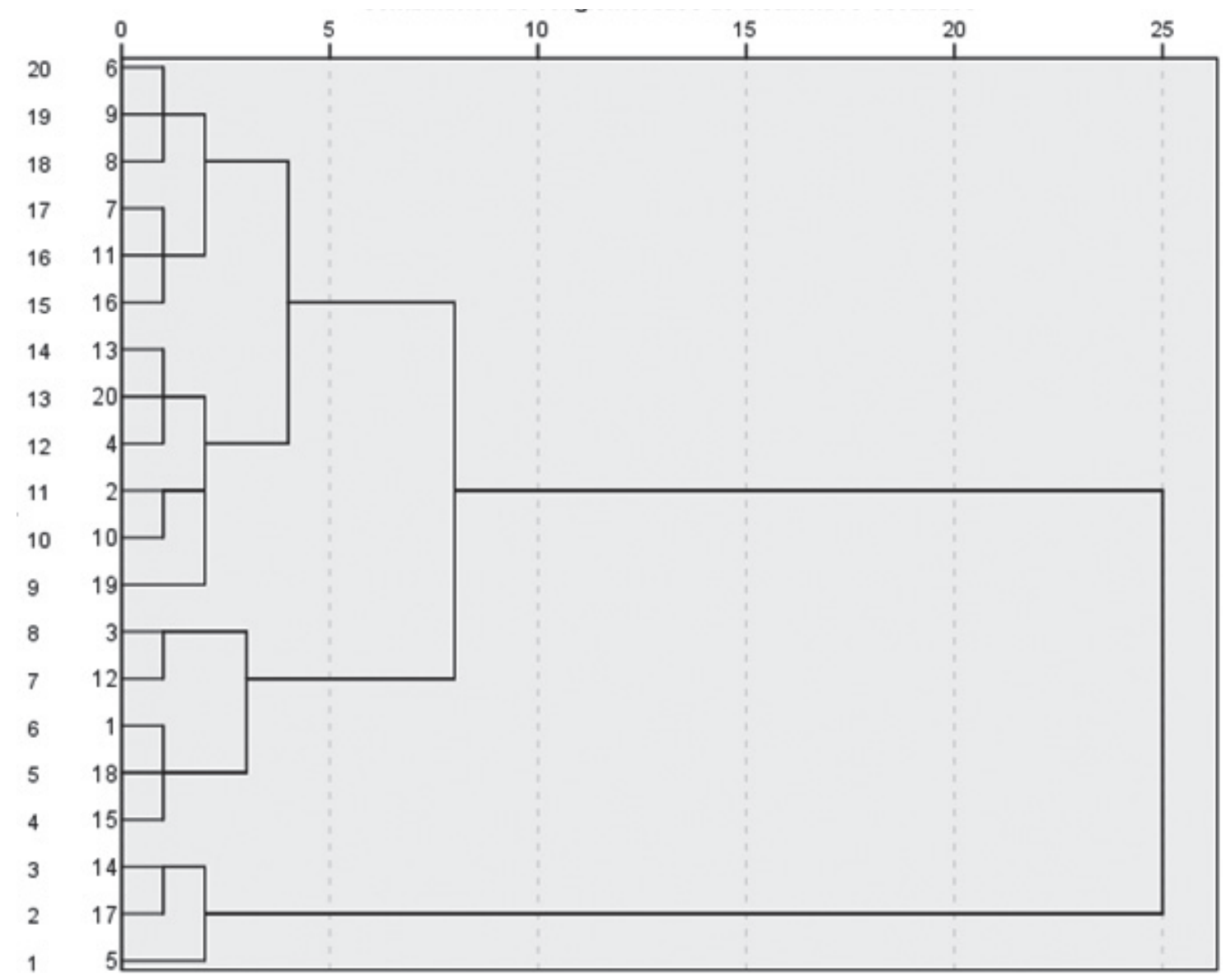

Figura 1. Dendrograma (vinculación de Ward). Combinación de conglomerados de distancia re-escalados.

Tabla 1. Diferencias en las dimensiones del EPT-C por cluster

\begin{tabular}{lccccc}
\hline $\begin{array}{c}\text { Dimensiones } \\
\text { EPT }\end{array}$ & Cluster & Media (DT) & $F$ & $p$ & $\eta_{\mathrm{p}}^{2}$ \\
\hline \multirow{3}{*}{ Atencional } & 1 & $30,00(4,08)^{\mathrm{a}}$ & & & \\
& 2 & $18,70(3,71)^{\mathrm{b}}$ & 12,477 & $0.000^{* * *}$ & .595 \\
& 3 & $23,50(4,04)^{\mathrm{b}}$ & & & \\
Operativa & 1 & $34,00(4,08)^{\mathrm{a}}$ & & & \\
& 2 & $25,10(3,28)^{\mathrm{b}}$ & 7,818 & $0.004^{* *}$ & .479 \\
& 3 & $28,33(4,50)^{\mathrm{a}, \mathrm{b}}$ & & & \\
Expresiva & 1 & $42,00(7,07)$ & & & \\
& 2 & $37,50(10,73)$ & 0,341 & 0,716 & - \\
& 3 & $38.67(7.15)$ & & & \\
Involucración & 1 & $26,25(9.57)$ & & & \\
& 3 & $26,50(4.30)$ & 0,061 & 0,941 & - \\
& 3 & $27,33(3.44)$ & & & \\
Instruccional & 2 & $29,70(7.04)$ & 1,859 & 0,186 & - \\
& 3 & $36,17(8.93)$ & & & \\
\hline
\end{tabular}

Nota. ${ }^{* * *} p<0.001 ; * * p 0.01$ Los superíndices a,b identifican diferencias post hoc (mismo superíndice significa ausencia de diferencia significativa entre grupos).
EPT por cluster. Como se puede observar, en las dimensiones vinculadas con las funciones predominantemente técnicas del EPT -atencional y operativa- se encontraron las diferencias significativas entre los perfiles. En las dimensiones atencional y operativa del EPT-C se obtuvieron diferencias estadísticamente significativas. Estas diferencias indicaron tamaños del efecto grandes en tres combinaciones de estilo personal de terapeutas representativas de diferentes posiciones para las dimensiones que operacionaliza el constructo. Así, los terapeutas del perfil 1 informaron una atención más focalizada en lo atencional y ser más pautados en lo operativo, los terapeutas del perfil 2 resultaron ser los menos focalizados y pautados, y los terapeutas del perfil 3 asumirían la posición intermedia o moderada en la dimensión operativa. Esta inferencia se basa en un examen visual de los perfiles.

A fines de establecer la magnitud de las asociaciones identificadas, así como precisar entre qué clusters se encuentra dicha asociación, se llevaron a cabo pruebas a posteriori en cada una de las variables dependientes cuya asociación respecto a los clusters fue estadísticamente significativa (dimensiones operativa y atencional). 


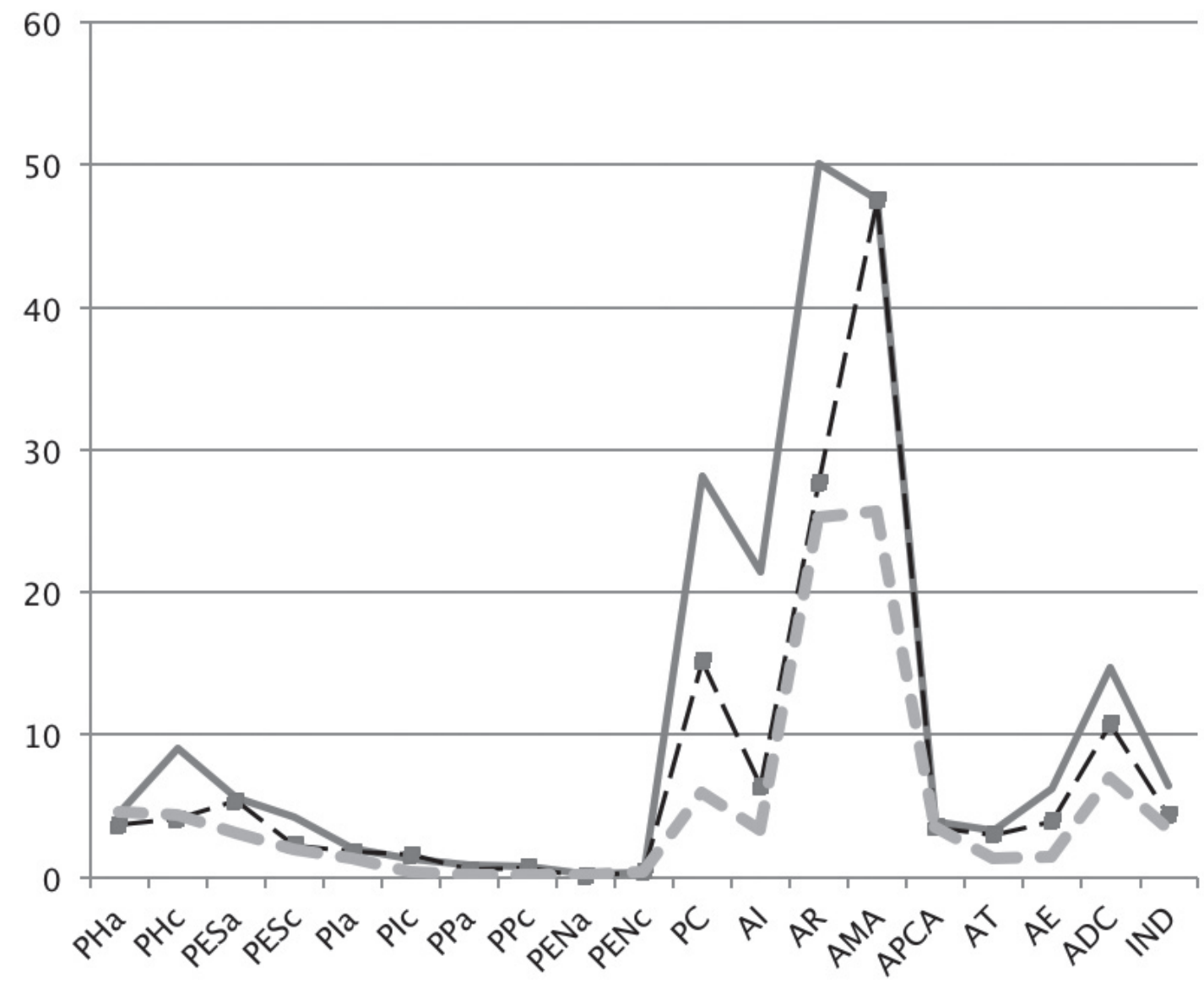

Cluster $1-$ Cluster $2-\infty$ Cluster 3

Figura 2. Frecuencia de intervenciones verbales según la GOTA-v por perfil terapéutico expresado en las medias da cada cluster.

Tal como se observa en la tabla 1 , se identificó un tamaño del efecto (eta cuadrado parcial) grande, según la clasificación de Harlow (2005), tanto en la escala operativa como atencional. En cuanto a la operativa, a partir de la prueba post hoc DHS Tukey se puede identificar que dicha significación estadística con una magnitud del efecto grande se presenta entre los perfiles 1 y $2(p<$ 0.05 ) es decir entre el cluster descripto como el más focalizado y más pautado y aquel que se identificó como menos focalizado y pautado. Esto se basó en el hecho que los terapeutas de ese cluster obtuvieron puntajes más altos en la dimensión atencional y operativa (más pautados) del EPT-C.

Por su parte, respecto a la dimensión atencional, las diferencias detectadas, también con una magnitud del efecto grande, se presentan entre el cluster 1 respecto al cluster $2(p<0.05)$ y al cluster $3(p<0.05)$. En otras palabras, entre el grupo caracterizado como más focalizado y pautado en comparación al menos focalizado y pautado, y también a aquel moderado en ambas dimensiones.

\section{Discusión}

El proceso de psicoterapia puede ser dividido en cuatro aspectos: contenido (qué se dice), acción (qué se hace), estilo (cómo se dice o hace) y calidad (cuán bien se dice o hace). Las categorías o dimensiones son referidos por los psicolingüistas y filósofos como actos de habla (Searle, 1969), y por los investigadores de procesos como modos de respuesta (Elliott et al., 1987). En este trabajo se cruzaron dos de esos aspectos: el estilo de los terapeutas con el contenido verbal de las sesiones. Se tomaron sesiones de cuatro pacientes diferentes por terapeuta para garantizar varianza en los resultados.

En la literatura de investigación en psicoterapia, tal como fue mencionado, existen antecedentes sobre el estudio de las acciones verbales de los terapeutas durante las sesiones y se ofrecen clasificaciones según diferentes criterios (Roussos et al., 2006). Uno de las primeras propuestas de clasificación fue la de Stiles (1992) en la que ya se consideraba la clasificación en diferentes actos de 
habla de Searle (1969). Una revisión detallada de este tema se puede consultar en Valdés et al. (2010) donde se citan 13 trabajos de los últimos 30 años. Algunos sistemas de clasificación incluyen a pacientes y terapeutas (Valdés et al., 2010). Los fundamentos y propiedades psicométricas de la GOTA-v fueron presentados por Fernández-Álvarez y et al. (2015). En este estudio se toman los resultados de la GOTA-v como criterio para el proceso de validación de otro instrumento que evalúa el Estilo Personal del Terapeuta: El EPT-C.

Los resultados aportan evidencia a favor de la validez concurrente del EPT-C con respecto a dos de sus dimensiones: la atencional y la operativa. Como fue formulado en las hipótesis de esta investigación, estas dos dimensiones operacionalizan las funciones del EPT-C, aspectos que, a diferencia de las otras tres, son factibles de verse reflejados en las conductas verbales de los terapeutas durante las sesiones. El EPT-C ha mostrado ser un instrumento confiable y válido, lo cual le aporta calidad técnica para su uso en contextos de investigación sobre la persona del terapeuta. En esa dirección, el establecimiento de la validez concurrente sobre la base de las observaciones de las intervenciones verbales, constituye un aporte relevante de este estudio.

A la hora de describir las características de los terapeutas de cada grupo, cabe destacar que aquellos incluidos en el cluster 1 son los más focalizados en su modo de prestar atención al material aportado por el paciente (dimensión atencional) y más pautados en su forma de intervenir (dimensión operativa). Esto permite postular de manera preliminar que se trata de terapeutas más $d i$ rectivos. Los terapeutas del cluster 2 resultan más abiertos en su foco atencional y menos pautados en sus intervenciones lo que permitiría identificarlos como no directivos en su comportamiento. El tercer cluster reúne terapeutas que se ubican en un lugar intermedio respecto a ambas dimensiones.

Otro aspecto a considerar en base a estos resultados es que, como puede observarse en la figura 2, todas las sesiones presentan una estructura similar en términos de frecuencia relativa por tipo de intervención. Todos los terapeutas utilizan preguntas, afirmaciones e indicaciones en una proporción similar en sus intervenciones. Sería de interés corroborar si esto se produce de manera similar a otros diálogos en contextos no terapéuticos o refleja alguna especificidad propia del discurso terapéutico.

Sin embargo, respecto al tipo de intervención, sí es posible identificar características específicas según los clusters, en particular en dos tipos de preguntas y en cuatro tipos de afirmaciones. Los terapeutas del cluster 1 realizan más preguntas cerradas por hechos $(\mathrm{PHc})$ que los otros dos, lo cual es compatible con la autodescrip- ción de ser terapeutas más directivos y focalizados en la medida de que las preguntas cerradas y por hechos indagan comportamientos, datos y hechos concretos, lo cual expresa la intención de orientar y guiar de un modo más explícito al paciente. Los terapeutas incluidos en el cluster 1 también realizan más preguntas de confirmación (PC) y aportan más información (AI) en comparación a los otros dos clusters.

En cuanto a las preguntas de confirmación y brindar más información, resultan modos de intervención coherentes desde el punto de vista teórico para un terapeuta directivo y focalizado, ya que en el proceso de orientar y guiar al paciente busca, por un lado, confirmar explícitamente que el paciente capte el contenido de la intervención y, por otro lado, dar más información como parte de una actitud proactiva y focalizada. Los terapeutas incluidos en el cluster 1 utilizaron más reflejos (AR) que los que reúnen los clusters 2 y 3 . Esta categoría no resulta, en principio, un aspecto directamente relacionado con una naturaleza más directiva, aunque podría ser de interés indagar cómo se presenta esta variable en otras muestras de terapeutas ni directivos ni focalizados. Este grupo de terapeutas, por último, presenta valores significativamente más elevados respecto al cluster 3, en las afirmaciones que van más allá de lo que dice el paciente (AMA) $\mathrm{y}$ en las afirmaciones que tienen que ver con el encuadre terapéutico (AE). En ambas categorías, del mismo modo que en las anteriores, resulta coherente que terapeutas más directivos intervengan brindando contenido que vaya más allá de lo expuesto por el terapeuta así como explicitando cuestiones vinculadas con el encuadre terapéutico. A su vez, los clusters 2 y 3 presentan diferencias significativas en relación a las preguntas por confirmación (PC) y en las afirmaciones que van más allá de lo que dicen los pacientes (AMA), siendo en ambos casos el cluster 3 el que presenta los valores inferiores. Estos datos sugieren formular que los terapeutas más directivos presentan valores más altos en algunas categorías de intervención verbal suponiendo que la naturaleza directiva implica más actividad por parte del terapeuta, mientras que los menos directivos expresan valores moderados en la frecuencia y tipo de intervenciones en sesión.

No se han podido establecer comparaciones con estudios similares debido a que en la literatura no se han encontrado antecedentes de instrumentos que evalúen el Estilo Personal del Terapeuta. Por otra parte, las otras taxonomías que se han propuesto para clasificar intervenciones utilizan algunas categorías comunes y otras diferentes. También incluyen algunas las respuestas de los pacientes a esas intervenciones. (Roussos, et al. 2006; Valdés et al. 2010). Por tal motivo no hay antecedentes de estudios en el que la clasificación del tipo de 
intervenciones durante las sesiones se utilice como criterio para validar un cuestionario que evalúe el EPT. El estudio presenta algunas limitaciones. En primer lugar, los resultados deben ser considerados preliminares. Como explica Klonsky (2014) uno de los aspectos problemáticos en la investigación en psicoterapia son los tamaños muestrales, del mismo modo que la obtención del material clínico (Taubner, Klasen y Munder, 2016). En el presente estudio, si bien se analizaron un número elevado de sesiones transcritas (79), que además provienen de un contexto clínico natural, los clusters finales quedan constituidos por pocos casos, y por lo tanto se trabaja de manera dependiente de la hipótesis de normalidad de las observaciones.

En cuanto a las líneas futuras de investigación, cabe destacar que para estudiar la validez concurrente de la función expresiva se está desarrollando la Guía Observacional de Terapeutas Aiglé en su modo no verbal. En este sentido, el tipo de preguntas del inventario que exploran algunas de las dimensiones del EPT-C no son observables durante la conducta en las sesiones, ya sea porque responden a aspectos de la actividad mental de los terapeutas o bien porque involucran facetas no verbales de la comunicación (como la prosodia, las posturas o los gestos corporales).

\section{Conflicto de intereses}

Los autores declaran que no existen conflictos de intereses.

\section{Referencias}

Baldwin, S. A., \& Imel, Z. E. (2013). Therapist variables in psychotherapy research. In M. J. Lambert, (Ed.), Bergin and Garfield's Handbook of Psychotherapy and Behavior Changef (6th ed., 258-297). New York, NY: Wiley.

Beutler, L. E., \& Forrester, B. (2014). What needs to change: Moving from «research informed» practice to «empirically effective» practice. Journal of Psychotherapy Integration, 24, 168177.

Beutler, L. E., \& Harwood, T. M. (2000). Prescriptive Psychotherapy. A Practical guide to systematic treatment selection. New York: Oxford

Beutler, L. E., Malik, M., Alimohamed, S., Harwood, T. M., Talebi, H., Noble, S., \& Wong, E. (2004). Therapist variables. In M. J. Lambert (Ed.), Handbook of psychotherapy and behavior change (5th ed.) (pp. 227-306). New York: John Wiley \& Sons.

Casari, L., Albanesi, S., Maristany, M., y Ison, M. (2016). Estilo personal del terapeuta en psicoterapeutas de adicciones. Revista Argentina de Clínica Psicológica, 25, 17-26.

Castañeiras, C., Ledesma, R., García, F., y Fernández-Álvarez, H. (2008). Evaluación del Estilo Personal del Terapeuta: presen- tación de una versión abreviada del Cuestionario EPT-C. Terapia Psicológica, 26, 5-13.

Corbella Santomá, S. (2002.) Compatibilidad entre el estilo personal del terapeuta y el perfil personal del paciente. Tesis doctoral. Facultat de Psicologia, Ciències de I'Educació i de I'Esport Blanquerna. Universitat Ramon Llull, Barcelona.

Crits-Christoph, P., Barancackie, K., Kurcias, J. S., Beck, A. T., Carroll, K., Perry, K., Luborsky, L., McLellan, A. T., Woody, G. E., Tompson, L., Gallagher, D., \& Zitrin, C. (1991). Meta-analysis of therapist effects in psychotherapy outcome studies. Psychotherapy Research, 1, 81-91.

Cuijpers, P., Ebert, D., Acarturk, C., Andersson, G., \& Cristea, I. A. (2016). Personalized Psychotherapy for Adult Depression: A Meta-Analytic Review. Behavior Therapy, 47, 966-980.

Elliott, R., Hill, C. E., Stiles, W. B., Friedlander, M. L., Mahrer, A., \& Margison, F. (1987). Primary therapist response modes: A comparison of six rating systems. Journal of Consulting and Clinical Psychology, 55, 218-223.

Fernández-Álvarez, J., García, F., Gómez, B., Lichtenberger, A. Wyss, G., \& Fernández-Álvarez, H. (2015). Looking into therapists' interventions during change processes. Panel en: Society for Psychotherapy Research, $46^{\text {th }}$ Annual International Meeting, Philadelphia, Pennsylvania, U.S.A.

Fernández-Álvarez, H., Castañeiras, C., Curtarelli, A., García, F., Gómez, B., Lichtenberger, A., y Corbella, S. (2015). Presentación de una Guía para la Observación y Clasificación de la Conducta Verbal de los Terapeutas. Terapia Psicológica, 33, 23-34.

Fernández-Álvarez, H., García, F., Lo Bianco, J., \& Corbella Santomá, S. (2003) Assessment Questionnaire on The Personal Style of the Therapist PST-Q. Clinical Psychology and Psychotherapy, 10, 116-125.

Fernández-Álvarez, H., García, F., \& Scherb, E. (1998). The Research Program at AIGLE. Journal of Clinical Psychology, 54, 343-359.

Fernández-Álvarez, H., Gómez, B., \& García, F. (2015). Bridging the gap between research and practice in a clinical and training network: Aigle's Program. Psychotherapy Research, 25, 84-94.

García, F., Castañeiras, C., y Fernández-Álvarez, H. (2008). La investigación en la clínica. En H. Fernández-Álvarez (Ed.) Integración y salud mental (pp.139-170). Bilbao: Desclée.

García, F., y Fernández-Álvarez, H. (2007). Investigación empírica sobre el Estilo Personal del Terapeuta: Una actualización. Revista Argentina de Clínica Psicológica, 16, 121-128.

García-Escalera, J., Chorot, P., Valiente, R. M., Reales, J. M., \& Sandín, B. (2016). Efficacy of transdiagnostic cognitive-behavioral therapy for anxiety and depression in adults, children and adolescents: A meta-analysis. Revista de Psicopatología y Psicología Clínica, 21, 147-175.

Gómez, B. (2015). El estilo personal del terapeuta en Psicooncología. Tesis doctoral. Universidad del Salvador, Buenos Aires.

Gómez, B., Castañeiras, C., Curtarelli, A., García, F., Ighani, M., Lichtenberger, A., \& Fernández-Álvarez, H. (2013). Verbal behavior in psychotherapy: similarities and differences in the way therapists of different orientation and experience level make statements. Poster en: Society for Psychotherapy Research $44^{\text {th }}$. Annual International Meeting, Brisbane, Australia. 
Gómez, B., Castañeiras, C., Curtarelli, A., García, F., M., Lichtenberger, A., \& Fernández-Álvarez, H. (2014). How do therapists ask questions? Revisited. Poster en: Society for Psychotherapy Research 45 ${ }^{\text {th }}$ Annual International Meeting, Copenhagen, Denmark.

Hill, C. E., \& O’Brien, K. (1999). Helping skills. Washington, DC: American Psychological Association.

Huppert, J. D., Kivity, Y., Barlow, D. H., Shear. M. K., Gorman, J. M., \& Woods. S. W. (2014). Therapist Effects and the Outcome-Alliance Correlation in Cognitive Behavioral Therapy for Panic Disorder with Agoraphobia. Behaviour Research and Therapy, 56, 26-34.

Klonsky, E. D. (2014). The future of empirically supported treatments: A comment on Coyne and Kok. Journal of Evidence-Based Psychotherapy, 14, 129-132.

Lutz, W., \& Barkham, M. (2015). Therapist effects. In R. Cautin y S. Lilienfeld (Eds.), Encyclopedia of clinical psychology (pp.1-6). Wiley-Blackwell.

Malik, M. L., Fleming, J., Penela, V., Harwood, M. T., Best, S., \& Beutler, L. (2002). Which therapist for which client? The interaction of therapist variation and client characteristics in affecting rates of change: An effectiveness study. Paper in: Society for Psychotherapy Research $33^{\text {th. }}$ Annual International Meeting, San Francisco, USA.

Nathan, P. E., \& Gorman, J. M. (Eds.). (2007). A guide to treatments that work (3rd ed.). New York: Oxford University Press.

Norcross, J. C., \& Lambert, M. J. (2014). Relationship science and practice in psychotherapy: Closing commentary. Psychotherapy (Chicago, Ill.), 51(3), 398-403.

Okiishi, J. C., Lambert, M. J., Eggett, D., Nielsen, L., Dayton, D. D., \& Vermeersch, D. A. (2006). An analysis of therapist treatment effects: Toward providing feedback to individual therapists on their clients' psychotherapy outcome. Journal of Clinical Psychology, 62, 1157-1172.
Rial, V., Castañeiras, C., García, F., Gómez, B., y Fernández-Álvarez, H. (2006). Estilo personal de terapeutas que trabajan con pacientes severamente perturbados: un estudio cuantitativo y cualitativo. Revista de la Asociación Española de Neuropsiquiatría, 26, 191-208.

Roth, A, \& Fonagy, P. What works for whom? A critical review of psychotherapy research. (2nd ed.). New York, NY: The Guilford Press; 2005.

Roussos, A. J., Etchebarne, I., y Waizmann, V. (2006). Un esquema clasificatorio para las intervenciones en psicoterapia cognitiva y psicoanalítica. Anuario de investigaciones, 13, 51-61.

Searle, J. (1969). Speech acts. An essay in the philosophy of language. Cambridge: Cambridge University Press.

Seligman, M. E. P. (1995). The effectiveness of psychotherapy: The consumer reports study. American Psychologist, 50, 965-974.

Stiles, W. B. (1992). Describing Talk. A Taxonomy of Verbal Response Modes. London: Sage Publications.

Taubner, S., Klasen, J., \& Munder, T. (2016). Why do psychotherapist participate in psychotherapy research and why not? Results of attitudes to Psychotherapy Research Questionnaire with a sample of experienced German psychotherapists. Psychotherapy Research, 16, 318-331.

Valdés, N., Tomicic, A., Pérez, J. C., y Krause, M. (2010). Sistema de codificación de la Actividad Terapéutica (scat-1.0): Dimensiones y categorías de las acciones comunicacionales de pacientes y psicoterapeutas. Revista Argentina de Clínica Psicológica, 19, 117-130.

Vázquez, L., y Gutiérrez de Vázquez, M. (2015). Orientación teórico-técnica y estilo personal del terapeuta. Revista Argentina de Clínica Psicológica, 24, 133-142.

Vega, E. (2006). El psicoterapeuta en neonatología: Rol y Estilo Personal. Buenos Aires: Lugar Editorial.

Wampold, B. E., \& Imel, Z. E. (2015). The great psychotherapy debate: the research evidence for what works in psychotherapy. New York: Routledge. 
Anexo I. Croquis de Glosario GOTA-v

\section{Preguntas}

1) $(\mathrm{PH})$ Preguntas sobre comportamientos, datos $y$ hechos

- Abierta

- Cerrada

2) (PES) Preguntas sobre la experiencia subjetiva

- Abierta

- Cerrada

a) Sobre pensamientos, emociones y evaluaciones del paciente sobre temas generales.

b) Sobre pensamientos, emociones y evaluaciones del paciente sobre la relación terapéutica.

c) Sobre las razones que da el paciente a distintos suceso

3) (PI) Preguntas para interpretar y relacionar

- Abierta

- Cerrada

a) Preguntas que sugieren hipótesis explicativas

b) Preguntas que sugieren relaciones explícitas entre temas

c) Preguntas a modo de reflexión sobre alternativas

d) Preguntas para confrontar

e) Preguntas para sugerir un curso de acción

4) (PP) Preguntas sobre lo que está sucediendo en el proceso terapéutico

- Abierta

- Cerrada

5) (PEN) Preguntas sobre el encuadre

- Abierta

- Cerrada

6) (PC) Preguntas de confirmación de recepción del mensaje

\section{Indicaciones}

1) (IND) Indicaciones

a) Sobre el encuadre

b) Indicaciones sobre el proceso terapéutico

c) Indicaciones sobre cursos de acción

\section{Afirmaciones}

1) (AI) Dar Información

a) De los procesos psicológicos normales / patológicos

b) Otra información

2) (AR) Retomar algo de lo que dice, escribe o habla el paciente
a) Reflejo
b) Completar la Frase
c) Recapitular
d) Paráfrasis
e) Señalamiento

3) (AMA) Ir Más Allá de lo que dice el paciente
a) Reformulación
b) Metáfora
c) Confrontación 

d) Predicción
e) Afirmación/Reflexiones para generar pensamientos alternativos.
f) Afirmaciones sobre sentimientos y acciones del paciente en sesión y respecto a la relación terapéutica.
g) Valoración

4) (APCA) Afirmaciones para promover cursos de acción

5) (AT) Afirmaciones sobre el terapeuta

6) (AP) Afirmaciones sobre el proceso terapéutico

a) Metaintervenciones

b) Sobre fases y etapas del tratamiento

c) Sobre medios y objetivos del tratamiento

d) Sobre el dispositivo y contexto del tratamiento

7) (AE) Afirmaciones respecto del encuadre

8) (ADC) Afirmaciones de confirmación. (ASC) Actos Sin Clasificar 